\title{
Editorial \\ Comparing the prevalence of rheumatic diseases in China with the rest of the world
}

\author{
David T Felson
}

Clinical Epidemiology Research \& Training Unit, 650 Albany Street, Suite X-200, Boston, MA 02118-2526, USA

Corresponding author: David T Felson, jendez@bu.edu

Published: 25 February 2008

Arthritis Research \& Therapy 2008, 10:106 (doi:10.1186/ar2369)

This article is online at http://arthritis-research.com/content/10/1/106

(C) 2008 BioMed Central Ltd

See related research by Zeng et al., http://arthritis-research.com/content/10/1/R17

\begin{abstract}
Geographic or ethnic differences in the occurrence of disease often provide insights into causes of disease and possible opportunities for disease prevention. Persons in China appear to have a consistently lower prevalence of rheumatoid arthritis and fibromyalgia than persons in the United States and Europe; reasons for these prevalence differences might include genetic differences, differences in environmental exposures or a combination of both. With increasing obesity, gout is becoming endemic in China. Finally, symptomatic knee osteoarthritis is extremely common in China and constitutes a major public health problem there.
\end{abstract}

In the present issue of Arthritis Research and Therapy Zeng and colleagues comprehensively review studies evaluating the prevalence of rheumatic disease in China [1]. Their report suggests variations in the prevalence of symptomatic osteoarthritis throughout China, consistent prevalences for ankylosing spondylitis and rheumatoid arthritis, an absence of fibromyalgia, and a temporal increase in the prevalence of gout. What should we make of all of these estimates and why are they important to us outside China?

Estimates of the prevalence of rheumatic disease provide information about the burden of disease and suggest a need for provision of health services. For knee osteoarthritis, where the prevalence in China is high and knee replacements are not widely available [2], there may be a need for additional provision of health services.

Comparing the prevalence of disease from one region with another often provides insights into disease etiology. For example, coronary heart disease rates increased as Japanese migrated from Japan to Hawaii to mainland United States, suggesting that diet and environment were major causes of coronary heart disease. For systemic lupus, which was not evaluated by Zeng and colleagues, a prevalence gradient exists with low prevalence of lupus occurring in Africans, moderate rates along the slave trade route among African descendents living in the Caribbean, and high rates in the United States [3]. Using data from the article by Zeng and colleagues, we can make inferences about differences in disease prevalence that might provide clues regarding rheumatic disease etiology.

Zeng and colleagues reviewed diverse studies, many of them using the Community Oriented Program for the Control of Rheumatic Diseases (COPCORD) protocol. The COPCORD has been a joint initiative by the World Health Organization and the International League of Associations for Rheumatology whose goal was to provide information about the epidemiology of rheumatic disease in developing countries. The COPCORD methods require comprehensive population-based sampling with a rigorous protocol translated into the language of interest, and then, for persons with rheumatic complaints, there is follow-up testing and often evaluation by a rheumatologist to determine how many persons have identifiable diseases.

Estimates of rheumatic disease derived from the COPCORD and other studies have varied and, despite the impressive standardization of this protocol, variations in prevalence rates are probably still due, in part, to differences in how the studies are carried out from site to site. Also, for rheumatoid arthritis, many of these studies only detected a few cases in a community, so the estimate of prevalence is accompanied by wide confidence bounds, and there was substantial variation in prevalence from community to community. Another variation source is that crude prevalence estimates are usually presented, whereas age-standardized prevalence would often be more informative, especially since rheumatic diseases vary in prevalence by age. An additional source of 
variation is that some of the studies may not sample large numbers of older people who tend to have the highest prevalence of many rheumatic diseases, including rheumatoid arthritis. Finally, participation rates may differ in each study. While most COPCORD studies have reported participation rates exceeding 80\% [4,5], some studies in Asia have not been so successful [6], and the failure to include persons with rheumatic diseases might seriously lower prevalence estimates.

Notwithstanding these limitations, the estimates by Zeng and colleagues suggest rheumatic disease prevalence rates in China that are different from those in the western hemisphere or Europe. Among these different rates is a prevalence of rheumatoid arthritis in China of roughly $0.2-0.3 \%$ of the population versus a prevalence of $0.8 \%$ in the western world [7]. The genes predisposing to rheumatoid arthritis may differ among Han Chinese versus mixed populations from the western world. Viral infectious triggers also could predominate in the West or could infect persons at an age when their response to infections was most likely to cause rheumatoid arthritis in genetically susceptible individuals, whereas similar individuals might be infected at a less susceptible age in China. Certainly, studies of Asian migrants to the western world might help determine whether Asians retain a lower prevalence of rheumatoid arthritis, or whether their rate after migration rises to that of the surrounding population, suggesting that environmental risk factors are the main cause of disease.

Zeng and colleagues report a prevalence of ankylosing spondylitis that is similar in China to reported estimates from the western world. In general, the prevalence of HLA-B27 in the community determines its prevalence of spondyloarthropathies. In the Han ethnic population HLA-B27 is present in between $4 \%$ and $6 \%$ of persons, a figure lower than that in Caucasians in the United States. This would be consistent with a lower prevalence of ankylosing spondylitis in China than the United States, rather than a comparable prevalence. Further documentation of this absence of a prevalence gradient should be confirmed with studies using broader definitions of disease (for example, undifferentiated spondyloarthropathy).

Gout has become more prevalent in China, a phenomenon also seen the United States in recent years. Causes include increasing rates of obesity and of aging in both populations, both risk factors strongly associated with the occurrence of gout. The dramatic increase in gout in China probably reflects the rapid westernization and urbanization of the society.

Finally, the absence of fibromyalgia in China could be explained by genetic differences in the processing of afferent nociceptors throughout the body or by the absence of central sensitization, a phenomenon thought to be closely tied to the development of fibromyalgia. Clearly some causes of fibromyalgia are sociocultural, and it is possible that the different cultural environment in China might affect the acceptability of reporting chronic generalized pain. Investigation of the fibromyalgia prevalence in migrant Chinese communities might provide insights into which factors account for the exceedingly low prevalence of fibromyalgia in China. In addition, given the import of genetic variations in explaining racial and ethnic differences in chronic pain [8,9], an assessment of the prevalence of these genetic abnormalities in persons of Han ancestry might be of interest.

In summary, Zeng and colleagues have comprehensively summarized the prevalence of different rheumatic diseases in China. Their results present a mosaic of rheumatic disease prevalence, which differs from the rheumatic disease distributions in the western world. For those of us from the West interested in better understanding the etiology of rheumatic disease, the prevalence gradients that can be inferred from these data regarding rheumatoid arthritis and fibromyalgia, both of which may be considerably less common in China than in the western world, might provide important insights into what causes these diseases.

\section{Competing interests}

The author declares that they have no competing interests.

\section{Acknowledgement}

The present work was supported by NIH AR47785.

\section{References}

1. Zeng Q, Chen R, Darmanwan J, Zheng YX, Chen SB, Wigley R, Chen SL, Zhang NZ: Rheumatic diseases in China. Arthritis Res Ther 2008, 10:R17.

2. Zhang Y, Xu L, Nevitt MC, Aliabadi P, Yu W, Qin M, Lui LY, Felson DT: Comparison of the Prevalence of Knee Osteoarthritis Between the Elderly Chinese Population in Beijing and Whites in the United States: The Beijing Osteoarthritis Study. Arthritis Rheum 2001, 44:2065-2071.

3. Bae SC, Fraser $\mathrm{P}$, Liang $\mathrm{MH}$ : The epidemiology of systemic lupus erythematosus in populations of African ancestry: a critical review of the 'prevalence gradient hypothesis'. Arthritis Rheum 1998, 41:2091-2099.

4. Dai SM, Han XH, Zhao DB, Shi YQ, Liu Y, Meng JM: Prevalence of rheumatic symptoms, rheumatoid arthritis, ankylosing spondylitis, and gout in Shanghai, China: a COPCORD study. J Rheumatol 2003, 30:2245-2251.

5. Haq SA, Darmawan J, Islam MN, Uddin MZ, Das BB, Rahman F, Chowdhury MA, Alam MN, Mahmud TA, Chowdhury MR, et al: Prevalence of rheumatic diseases and associated outcomes in rural and urban communities in Bangladesh: a COPCORD study. J Rheumatol 2005. 32:348-353.

6. Chou, CT, Pei L, Chang DM, Lee CF, Schumacher HR, Liang MH: Prevalence of rheumatic diseases in Taiwan: a population study of urban, suburban, rural differences. J Rheumato/ 1994, 21:302-306.

7. Silman A, Hochberg MC: Epidemiology of the Rheumatic Diseases. 2nd edition. New York: Oxford University Press; 2001.

8. Kim, H, Neubert JK, San Miguel A, Xu K, Krishnaraju RK, ladarola MJ, Goldman D, Dionne RA: Genetic influence on variability in human acute experimental pain sensitivity associated with gender, ethnicity and psychological temperament. Pain 2004, 109:488-496.

9. Diatchenko, L, Slade GD, Nackley AG, Bhalang K, Sigurdsson A Belfer I, Goldman D, Xu K, Shabalina SA, Shagin D, et al: Genetic basis for individual variations in pain perception and the development of a chronic pain condition. Hum Mol Genet 2005, 14:135-143. 